\title{
Study of cross talk between phosphatases and OGA on a ZO-3-derived peptide
}

\author{
Suhela Sharif ${ }^{1} \cdot$ Jie Shi $^{1} \cdot$ Rob Ruijtenbeek $^{1,2} \cdot$ Roland J. Pieters $^{1}$ (D
}

Received: 17 September 2018 / Accepted: 11 January 2019 / Published online: 6 February 2019

(c) The Author(s) 2019

\begin{abstract}
O-GlcNAcylation, like phosphorylation, is a dynamic and rapid posttranslational modification which regulates many cellular processes. Phosphorylation on tyrosine and O-GlcNAcylation on nearby serine or threonine residues may modulate each other. Indeed, by using a microarray with a peptide model system based on the ZO-3 protein, extensive cross talk between O-GlcNAcylation by OGT and phosphorylation by kinases was observed. However, studying the effects of kinases and OGT without the reverse processes catalyzed by phosphatases and O-GlcNAcase (OGA) does not provide a complete picture of the cross talk. The study of the missing part showed that nearby phosphorylation affects the de-O-GlcNAcylation by OGA, but not to the same extent as it affects the O-GlcNAcylation by OGT. Both the phosphorylation and de-phosphorylation processes were only slightly affected by the presence of an O-GlcNAc residue on a nearby serine.
\end{abstract}

Keywords O-GlcNAc $\cdot$ Peptide microarray $\cdot$ Phosphatase $\cdot$ O-GlcNAcase $\cdot$ Cross talk $\cdot$ Zonula-occludens-3 (ZO-3)

\section{Introduction}

Posttranslational modifications (PTMs) of proteins play an important role in cell signaling and regulation of gene expression or protein expression (Prabakaran et al. 2012). Some proteins are modified by two or more PTMs that can affect each other and regulate each other's activity (Venne et al. 2014). One example of this is the cross talk between O-GlcNAcylation and phosphorylation. O-GlcNAcylation, like phosphorylation, is a dynamic and rapid posttranslational modification (PTM) and directly regulates many cellular processes (Hart et al. 2011). However, unlike phosphorylation, which is controlled by a large family of kinases and phosphatases specific to each substrate, only two enzymes, O-GlcNAc transferase (OGT) and O-GlcNAcase (OGA),

Handling Editor: K. Barnouin.

Roland J. Pieters

R.J.Pieters@uu.nl

1 Department of Chemical Biology and Drug Discovery, Utrecht Institute for Pharmaceutical Sciences, Utrecht University, P. O. Box 80082, 3508 TB Utrecht, The Netherlands

2 PamGene International BV, 's-Hertogenbosch, The Netherlands control this modification regardless of substrate (Zachara et al. 2004). A $\beta$ - $N$-acetylglucosamine (O-GlcNAc) is added to the serine or threonine residues of a protein by O-GlcNAc transferase (OGT). O-GlcNAcase (OGA) reverses the O-GlcNAcylation process by removal of O-GlcNAc from the proteins.

O-GlcNAcylation and phosphorylation can happen on the same serine or threonine amino acids (Wang et al. 2008) or on amino acids proximal to each other (Cheng et al. 2000). Therefore, these modifications have the potential to compete with and regulate each other. A few studies have indicated an interplay between serine/threonine phosphorylation and O-GlcNAcylation and its effect on signaling transduction (Butkinaree et al. 2010), transcription (Baldini et al. 2016) and with possible relevance to diseases such as diabetes, cancer and Alzheimer disease (Bond and Hanover 2013).

The mapping of O-GlcNAcylation sites in proteins using mass spectrometry revealed that in addition to the above interplay involving serine and threonine phosphorylation (Leney et al. 2017; van der Laarse et al. 2018), there may also be cross talk between O-GlcNAcylation and tyrosine phosphorylation (Mishra et al. 2011; van der Laarse et al. 2018).

While studying this phenomenon in a biological system is challenging, the use of a microarray with peptide sequences derived from substrate proteins has proven valuable and 
effective for both kinase (Sikkema et al. 2009) and O-GlcNAcylation studies (Shi et al. 2016). A recent study in our group using such a microarray analysis (Shi et al. 2017) showed that from hundreds of tyrosine kinase substrates, the peptide ZO3_357_371 was identified as a dual substrate. It is derived from the $\mathrm{ZO}-3$ protein which has been shown to be associated with a poor prognosis in patients with breast cancer (Martin et al. 2004), and can both be phosphorylated on Tyr 364 by the JAK2 kinase and O-GlcNAcylated on nearby Ser369 by OGT. This system allowed us to study the cross talk between the processes which showed that phosphorylation at Tyr364 dramatically slowed down the O-GlcNAcylation at Ser369, while O-GlcNAcylation at Ser369 had no effect on phosphorylation of Tyr364.

The missing part of the story, however, was: how does the phosphate group affect the de-O-GlcNAcylation by OGA and how does the O-GlcNAc group affect the dephosphorylation by phosphatases? To this end, we synthesized the ZO-3 peptide RESSYDIY(p)RVPSS(g)QS, which contains both the phosphate and the O-GlcNAc group (Fig. 1a). To find a phosphatase that functions with phosphorylated ZO-3, we tested human cytoplasmic protein tyrosine phosphatase 1B (PTP1B), alkaline phosphatase (AP), and the Src homology 2-containing protein tyrosine phosphatases (SHIP1 and SHIP2). Using an activity-based microarray (Sharif et al. 2017), we studied the effect of the phosphate and O-GlcNAc groups on OGA and four selected phosphatases. Our results revealed that the O-GlcNAc group on Ser369, has a small effect on the removal of the phosphate group on Tyr364 by the phosphatases. On the other hand, phosphorylation of Tyr364 significantly hinders the removal of O-GlcNAc by OGA.

\section{Materials and methods}

\section{Materials}

Fmoc-Tyr $\left(\mathrm{PO}\left(\mathrm{NMe}_{2}\right)_{2}\right)-\mathrm{OH}$ was purchased from Merck Millipore. Fmoc-Ser( $\left(\beta\right.$-D-GlcNAc $\left.(\mathrm{Ac})_{3}\right)-\mathrm{OH}$ was synthesized in our laboratory according to a literature procedure (Sharif et al. 2017). Primary antibody mouse monoclonal Anti-OLinked N-Acetylglucosamine antibody [RL2] (ab2739) was purchased from Abcam (London, England) and FITC labeled goat anti-mouse secondary antibody was obtained from Thermo Scientific (Bleiswijk, the Netherlands). PamChip4 microarray chips, fluorescein-labeled PY20 antibody, PTP 1B, AP, SHIP1, SHIP2 and PTP buffer were provided by PamGene (Pamgene International B.V., 's-Hertogenbosch, The Netherlands). O-GlcNAcase (OGA) was overexpressed as previously reported (Sharif et al. 2017). (a)

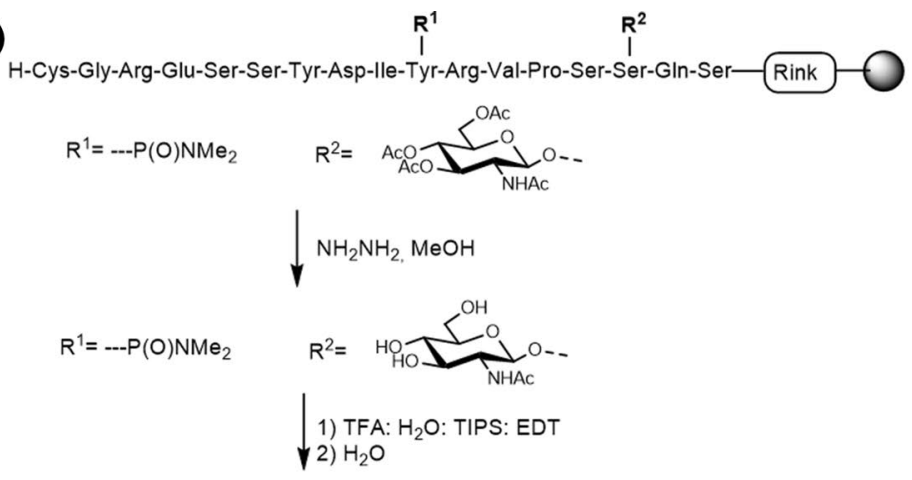

Y364p,S268g-ZO3: $\quad \mathrm{R}^{1}=$-.-P(O)OH $\mathrm{OH}_{2} \quad \mathrm{R}^{2}=$

other peptides:

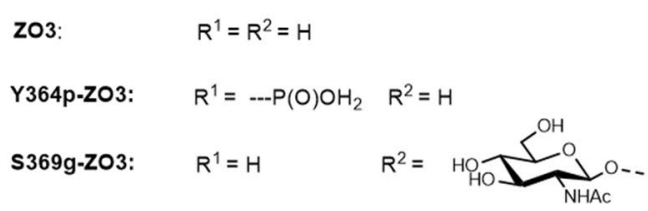

(b)

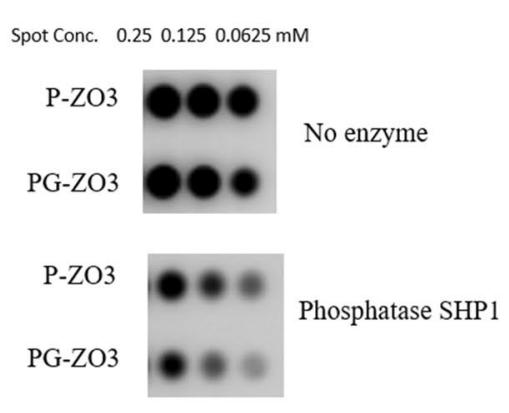

Detection: FITC labeled anti- pY- antibody (PY20)

Fig. 1 a Solid phase synthesis of derivatized ZO3-peptide RESSYDIY(p)RVPSS(g)QS and the structures of the related ZO3-peptides. b PY20 antibody staining of P-ZO3 and PG-ZO3 (top) and the degree of phosphorylation after the action of phosphatase SHP1 after $3 \mathrm{~h}$ 


\section{Solid-phase peptide synthesis, purification and LC- MS analysis}

The peptides RESSYDIYRVPSSQS, RESSYDIY(p) RVPSSQS and RESSYDIYRVPSS(g)QS were synthesized for a recent study in our group (Shi et al. 2017). Synthesis of the peptide RESSYDIY(p)RVPSS(g)QS was performed by following a standard Fmoc SPPS strategy with a symphony multiple peptide synthesizer (Protein Technology, Tucson, AZ, USA) and starting from a Rink amide resin (Sharif et al. 2017) (Fig. 1a). To synthesize this peptide, the Fmoc amino acids, an Fmoc-Ser( $\beta$-D-GlcNAc(Ac) $\left.)_{3}\right)-\mathrm{OH}$ and Fmoc$\operatorname{Tyr}\left(\mathrm{PO}\left(\mathrm{NMe}_{2}\right)_{2}\right)-\mathrm{OH}$ were used. To deprotect the peptide, $20 \%$ piperidine in DMF was used. The coupling was carried out using 1:0.9:2 amino acid/HBTU/DIPEA in DMF. Hydrazine in methanol $(80 \%)$ was added to the resin for acetyl deprotection of O-GlcNAc. The resins were then incubated with cleavage mixture including TFA (trifluoroacetic acid): $\mathrm{H}_{2} \mathrm{O}$ :tri-isopropylsilane (TIPS):1, 2-ethanedithiol (EDT) (9:0.5:0.25:0.25, v/v/v/v, $10 \mathrm{~mL})$ and agitated for $4 \mathrm{~h}$ at room temperature under a nitrogen atmosphere. Deprotection of phosphotyrosine was performed by the addition of $10 \%$ by volume of water into the cleavage mixture and then stirred overnight at room temperature (Fig. 1a). After filtration of the cleavage mixture, the resin was washed with TFA and DCM. The peptide was precipitated using precooled diethyl ether and obtained by centrifugation. Purification was carried out on a preparative reverse phase HPLC on an Applied Biosystems 400 Solvent Delivery system with a Luna C8 column $(250 \times 21.20 \mathrm{~mm} / 10 \mu$ micro; Phenomenex $)$.

After purification, the product showed the correct mass (expected for $\mathrm{C}_{88} \mathrm{H}_{141} \mathrm{~N}_{26} \mathrm{O}_{37} \mathrm{SP}(\mathrm{M}+2 \mathrm{H})^{2+}=1108.47$, found: 1108.85 ) and $100 \%$ purity as determined by HPLC (detection simultaneously at 214, $254 \mathrm{~nm}$ and evaporative

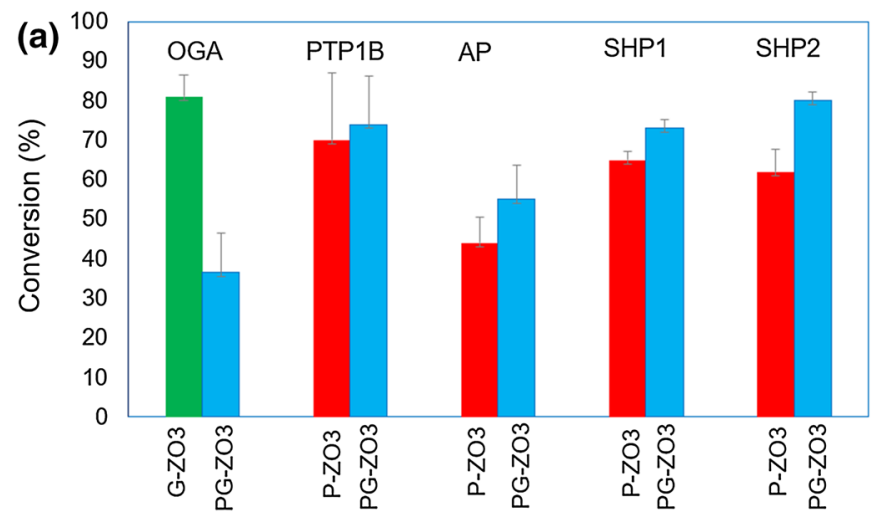

Fig. 2 a Degree of conversion of G-ZO3, P-ZO3 and PG-ZO3 by the respective enzymes relative to a no-enzyme reaction. For OGA, this means O-GlcNAc removal and for the rest de-phosphorylation. The average of these percentages was used. Error bars indicate the SEM $(N=3)$. b Scheme of the possible states of O-GlcNAcylation and light scattering detection) equipped with an analytical $\mathrm{C}_{8}$ column in combination with buffers $\mathrm{A}: \mathrm{H}_{2} \mathrm{O}, \mathrm{B}$ : $\mathrm{MeCN}$, and $\mathrm{A}$ and $\mathrm{B}$ with $0.1 \%$ aqueous trifluoroacetic acid (TFA) coupled with ESI-MS.

\section{Microarray assay}

The synthesized peptides were immobilized at different spotting concentrations on PamChip4 microarray chips (Pamgene International B.V., 's-Hertogenbosch, The Netherlands). The arrays were first blocked by $1 \%$ BSA. The OGA buffer (Britton-Robinson buffer $\mathrm{pH}$ 7) with/without OGA and PTP buffer (50 mM Tris/ $\mathrm{HCl} \mathrm{pH} \mathrm{7.5,} 10 \mathrm{mM} \mathrm{MgCl}_{2}$, $1 \mathrm{mM}$ EGTA, $2 \mathrm{mM}$ DTT, $0.01 \%$ Brij 35) with/without phosphatases was added to the arrays. After $3 \mathrm{~h}$ incubation, the appropriate antibody was added to the arrays to determine the degree of de-functionalization.

\section{Results}

The effect of phosphorylated Tyr364 on the removal of the O-GlcNAc moiety from the O-GlcNAcylated Ser369 of the ZO-3 peptide by OGA was studied with the peptide RESSYDIY(p)RVPSS(g)QS on the microarray. The removal of the O-GlcNAc moiety from the O-GlcNAcylated Ser369 leads to a decrease in signal intensity which is derived from the anti-O-GlcNAc antibody intensity (intensity is set at $100 \%$ when no enzyme is present). A high activity of the OGA enzyme thus leads to a low signal intensity. The OGA activity on O-GlcNAcylated RESSYDIYRVPSS(g)QS was stronger than that on the peptide RESSYDIY(p)RVPSS(g) QS that is additionally phosphorylated (Fig. 2a). The degree of O-GlcNAc removal of the doubly functionalized peptide

(b)

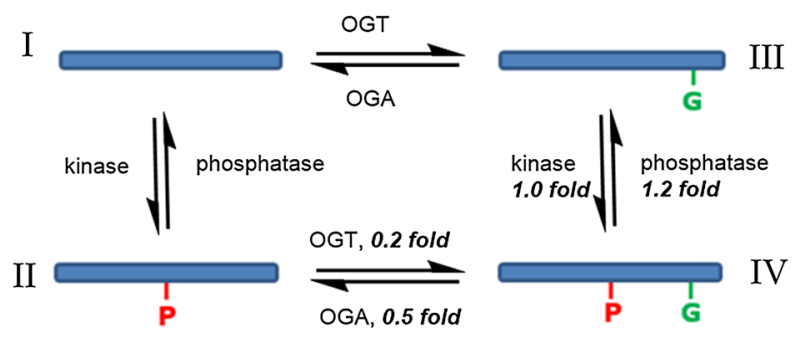

phosphorylation of the ZO-3 peptide and the conversion between the states, by the four types of enzymes. The degrees of functionalization to and from state IV (PG-ZO3) are compared to those to and from state I (ZO3) 
was ca. $50 \%$ in comparison to that of the mono-functionalized peptide (ca. $40 \%$ vs $80 \%$ removal).

Similarly, the effect of O-GlcNAcylated Ser369 on the removal of the phosphate moiety by phosphatase PTP 1B from the phosphorylated Tyr364 of RESSYDIY(p) RVPSS(g)QS peptide was determined. Here, the removal of the phosphate moiety from the phosphorylated Tyr 364 led to a decrease in signal intensity which is derived from the anti-tyrosine phosphate antibody PY20. The PTP 1B phosphatase showed essentially no difference in the degree of dephosphorylation on the doubly functionalized RESSYDIY(p)RVPSS(g)QS peptide compared to the merely phosphorylated RESSYDIY(p)RVPSSQS. In both cases, ca. $70 \%$ of the phosphates were removed (Figs. 1b, 2a).

In addition to PTP $1 \mathrm{~B}$, different tyrosine phosphatases were also used. These were AP, SHIP1 and SHIP2. The results for these phosphatases were similar to that of PTP $1 \mathrm{~B}$ and all of them showed little difference between the two substrates. End points showed higher degrees of dephosphorylation when an O-GlcNAc residue was also present, but the effects were no bigger than ca. 1.3-fold (Fig. 2a).

\section{Discussion}

Our recent study on the potential cross talk between O-GlcNAcylation and tyrosine phosphorylation using an activity-based peptide microarray showed that the phosphorylation of a tyrosine in these peptides inhibited their subsequent O-GlcNAcylation by OGT. However, vice versa, the O-GlcNAcylation of these peptides had no effect on their subsequent tyrosine phosphorylation. A specific peptide (ZO3_357_371) was used in that study representing a rare case of a dual known substrate (Shi et al., 2017). The effects were seen with both antibody-based detection and also with UDP or ADP-glo assays that report on ADP or UDP production in phosphorylation and O-GlcNAcylation. Having validated these tools, we here used the antibodybased detection for the reverse reactions determining the relative disappearance of the signal. The present study of cross talk of the reverse reactions was aimed to complete the picture of how both modifications affect each other in this model system. Phosphorylated Tyr364 was found to slightly hinder the removal of the O-GlcNAc on Ser369 moiety by OGA which leads to a ca 50\% reduced O-GlcNAc removal from the difunctionalized peptide. An effect of the nearby O-GlcNAc was a slightly enhanced de-phosphorylation by the four phosphatases used, i.e., PTP 1B, AP, SHIP1 and SHIP2 of a factor 1.2 on average, compared to the previously measured effect of the phosphate on O-GlcNAcylation by OGT which was at least a factor of 4 . Both effects observed here were relatively small.
Based on this, some scenarios can be envisaged that can be seen in Fig. 2b. In general, a high nutrition situation will result in a higher degree of O-GlcNAcylation such as III. Similarly, cells can be in a situation of high kinase activity which will lead to II. If both conditions apply, situation IV may be dominant and if neither applies situation I. It is clear how the two modifications can influence each other. If, e.g., there is a high kinase situation (II) and nutrient levels increase, this will lead to a relatively low level of O-GlcNAcylation than would otherwise be the case because of the strongly reduced rate of O-GlcNAcylation of phosphorylated peptides (i.e., the conversion of II-IV).

We may not yet be able to correlate the results of our model studies to those on critical proteins in vivo or disease states, but considering that a disease like cancer depends critically on a delicate balance of both phosphorylation and nutrient levels, the interplay as noted here may be of importance. These results provide some insights into the cross talk between O-GlcNAcylation and tyrosine phosphorylation and we speculate that the $\mathrm{ZO} 3$ results may be more general. Tyrosine phosphorylation and O-GlcNAcylation may have a significant contribution to the dynamic regulation of each other.

OpenAccess This article is distributed under the terms of the Creative Commons Attribution 4.0 International License (http://creativeco mmons.org/licenses/by/4.0/), which permits unrestricted use, distribution, and reproduction in any medium, provided you give appropriate credit to the original author(s) and the source, provide a link to the Creative Commons license, and indicate if changes were made.

\section{References}

Baldini SF, Wavelet C, Hainault I et al (2016) The nutrient-dependent O-GlcNAc modification controls the expression of liver fatty acid synthase. J Mol Biol 428:3295-3304. https://doi.org/10.1016/j. jmb.2016.04.035

Bond MR, Hanover JA (2013) O-GlcNAc cycling: a link between metabolism and chronic disease. Annu Rev Nutr 33:205-229. https://doi.org/10.1146/annurev-nutr-071812-161240

Butkinaree C, Park K, Hart GW (2010) O-linked beta- $N$-acetylglucosamine (O-GlcNAc): extensive crosstalk with phosphorylation to regulate signaling and transcription in response to nutrients and stress. Biochim Biophys Acta Gen Sub 1800:96-106. https://doi. org/10.1016/j.bbagen.2009.07.018

Cheng X, Cole RN, Zaia J, Hart GW (2000) Alternative $\mathrm{O}$-glycosylation/O-Phosphorylation of the murine estrogen receptor $\beta \dagger$. Biochemistry 39:11609-11620. https://doi.org/10.1021/ bi000755i

Hart GW, Slawson C, Ramirez-Correa G, Lagerlof O (2011) Cross talk between O-GlcNAcylation and phosphorylation: roles in signaling, transcription, and chronic disease. Annu Rev Biochem 80:825-858. https://doi.org/10.1146/annurev-biochem-06060 8-102511

Leney AC, El Atmioui D, Wu W et al (2017) Elucidating crosstalk mechanisms between phosphorylation and O-GlcNAcylation. 
Proc Natl Acad Sci 114:E7255-E7261. https://doi.org/10.1073/ pnas. 1620529114

Martin TA, Watkins G, Mansel RE, Jiang WG (2004) Loss of tight junction plaque molecules in breast cancer tissues is associated with a poor prognosis in patients with breast cancer. Eur J Cancer 40:2717-2725. https://doi.org/10.1016/j.ejca.2004.08.008

Mishra S, Ande SR, Salter NW (2011) O-GlcNAc modification: why so intimately associated with phosphorylation? Cell Commun Signal 9:1-4. https://doi.org/10.1186/1478-811X-9-1

Prabakaran S, Lippens G, Steen H, Gunawardena J (2012) Posttranslational modification: nature's escape from genetic imprisonment and the basis for dynamic information encoding. Wiley Interdiscip Rev Syst Biol Med 4:565-583. https://doi.org/10.1002/ wsbm. 1185

Sharif S, Shi J, Bourakba M et al (2017) Measuring O-GlcNAc cleavage by OGA and cell lysates on a peptide microarray. Anal Biochem 532:12-18. https://doi.org/10.1016/j.ab.2017.05.027

Shi J, Sharif S, Ruijtenbeek R, Pieters RJ (2016) Activity based highthroughput screening for novel O-GlcNAc transferase substrates using a dynamic peptide microarray. PLoS One 11:e0151085. https://doi.org/10.1371/journal.pone.0151085

Shi J, Tomašič T, Sharif S et al (2017) Peptide microarray analysis of the cross-talk between O-GlcNAcylation and tyrosine phosphorylation. FEBS Lett 591:1872-1883. https://doi. org/10.1002/1873-3468.12708
Sikkema AH, Diks SH, den Dunnen WFA et al (2009) Kinome profiling in pediatric brain tumors as a new approach for target discovery. Cancer Res 69:5987-5995. https://doi.org/10.1158/00085472.CAN-08-3660

van der Laarse SAM, Leney AC, Heck AJR (2018) Crosstalk between phosphorylation and O-GlcNAcylation: friend or foe. FEBS J 285:3152-3167. https://doi.org/10.1111/febs.14491

Venne AS, Kollipara L, Zahedi RP (2014) The next level of complexity: crosstalk of posttranslational modifications. Proteomics 14:513-524. https://doi.org/10.1002/pmic.201300344

Wang Z, Gucek M, Hart GW (2008) Cross-talk between GlcNAcylation and phosphorylation: site-specific phosphorylation dynamics in response to globally elevated O-GlcNAc. Proc Natl Acad Sci USA 105:13793-13798. https://doi.org/10.1073/pnas.0806216105

Zachara NE, O'Donnell N, Cheung WD et al (2004) Dynamic O-GlcNAc modification of nucleocytoplasmic proteins in response to stress: a survival response of mammalian cells. J Biol Chem 279:30133-30142. https://doi.org/10.1074/jbc.M403773200

Publisher's Note Springer Nature remains neutral with regard to jurisdictional claims in published maps and institutional affiliations. 